\title{
Recent Progress in the Computational Many-Body Theory of Metal Surfaces
}

\author{
Maziar Nekovee ${ }^{1}$ and J. M. Pitarke ${ }^{2,3}$ \\ ${ }^{1}$ Centre for Computational Science, Queen Mary and Westfield College, University of London, \\ Mile End Road, London E1 $4 \mathrm{NS}$, UK \\ ${ }^{2}$ Materia Kondentsatuaren Fisika Saila, Zientzi Fakultatea, Euskal Herriko Unibertsitatea, \\ 644 Posta kutxatila, 48080 Bilbo, Basque Country, Spain \\ ${ }^{3}$ Donostia International Physics Center (DIPC) and Centro Mixto CSIC-UPV/EHU, \\ Donostia, Basque Country, Spain
}

(July 30, 2021)

\begin{abstract}
In this article we describe recent progress in the computational many-body theory of metal surfaces, and focus on current techniques beyond the local-density approximation of density-functional theory. We overview various applications to ground and excited states. We discuss the exchangecorrelation hole, the surface energy, and the work function of jellium surfaces, as obtained within the random-phase approximation, a time-dependent density-functional approach, and quantum Monte Carlo methods. We also present a survey of recent quasiparticle calculations of unoccupied states at both jellium and real surfaces.
\end{abstract}

\section{INTRODUCTION}

Prerequisite for calculating the electronic properties of solid surfaces is a practical procedure for the treatment of electron-electron (e-e) interactions in large, strongly inhomogeneous electron systems. Over the last three decades, almost all first-principles electronic-structure calculations have been carried out within density-functional theory (DFT) , which solves the many-electron problem by introducing an auxiliary non-interacting system with the same electron density 2 . Although DFT is a formally exact theory, it relies on the knowledge of the so-called exchangecorrelation (xc) energy as a functional of the electron density. The best known approximation for this functional is the local-density approximation (LDA)2, which has proved remarkably successful in various applications to ground-state properties of solid surfaces $B$. However, this simple approximation presents two important shortcomings. First, the LDA is unable to provide the highly accurate total energies that are required in the application of DFT to chemical reactions at surfaces. Secondly, it yields an inaccurate description of the long-range xc-hole density at solid surfaces, and fails to reproduce the correct image-like asymptotic behaviour of the surface barrier, which plays an important role in the interpretation of a number of surface-sensitive experiments. Furthermore, the knowledge of the finite quasiparticle lifetimes of unoccupied states is beyond the scope of DFT.

The aim of this article is to survey recent computational work on the description of many-body effects in both ground- and excited-state properties of metal surfaces. In section II we give a short overview of the state-of-the-art computational many-body theories of the inhomogeneous electron gas: DFT, in the LDA and its various gradientcorrected forms, quantum Monte Carlo (QMC) methods, and Green function theory in the so-called GW approximation. In section III we focus on a description of ground-state properties of metal surfaces, and we critically examine the performance of current many-body schemes in the computation of two of the most fundamental magnitudes, namely the surface energy and the work function. In section IV we examine quasiparticle calculations of the surface barrier and the binding energies and lifetimes of image-potential induced states. These so-called image states 5 form a Rydberg-like series, which converges towards the vacuum energy, and their existence is perhaps the most striking manifestation of long-range many-body effects at surfaces. We conclude this article in section $\mathrm{V}$ with some perspectives for future research in this area.

We use Hartree atomic units throughout, unless otherwise specified. In these units, $e=m_{e}=\hbar=4 \pi \epsilon_{0}=1$.

\section{THEORY OF THE INHOMOGENEOUS ELECTRON GAS}

Under the assumption that electronic and ionic degrees of freedom can be decoupled, the problem of $N$ electrons in a solid can be described by the following Hamiltonian:

$$
\hat{H}=\sum_{i=1}^{N}\left[-\frac{1}{2} \nabla_{i}^{2}+V_{e x t}\left(\mathbf{r}_{i}\right)\right]+\sum_{i<j}^{N} \frac{1}{\left|\mathbf{r}_{i}-\mathbf{r}_{j}\right|} .
$$


Here $\mathbf{r}_{i}$ is the coordinate of electron $i$, and $V_{\text {ext }}\left(\mathbf{r}_{i}\right)$ is the external potential accounting for the interaction with the fixed atomic nuclei.

\section{A. Density-functional theory}

DFT is a formally exact theory based on two theorems by Hohenberg and Kohnt The first theorem is extraordinary: it states that the ground-state density $n(\mathbf{r})$ of a system of interacting electrons determines the ground-state properties of the system uniquely. The second theorem states that the ground-state energy can be obtained by minimising an energy functional $E[\tilde{n}(\mathbf{r})]$ of the electron density. The minimum value of $E[\tilde{n}(\mathbf{r})]$ is the exact ground-state energy, and is attained when $\tilde{n}(\mathbf{r})$ is the exact ground-state density $n(\mathbf{r})$.

In the Kohn-Sham formulation of DFT2, the ground-state density can be obtained by calculating the eigenfunctions $\phi_{i}(\mathbf{r})$ and eigenvalues $\varepsilon_{i}$ of non-interacting, single-particle equations, yielding

$$
n(\mathbf{r})=\sum_{i=1}^{N}\left|\phi_{i}(\mathbf{r})\right|^{2},
$$

and the energy functional is written in the form

$$
E[\tilde{n}(\mathbf{r})]=T_{0}[\tilde{n}(\mathbf{r})]+\int d \mathbf{r} \tilde{n}(\mathbf{r}) V_{e x t}(\mathbf{r})+\frac{1}{2} \int d \mathbf{r} \frac{\tilde{n}(\mathbf{r}) \tilde{n}\left(\mathbf{r}^{\prime}\right)}{\left|\mathbf{r}-\mathbf{r}^{\prime}\right|}+E_{x c}[\tilde{n}(\mathbf{r})]
$$

Here $T_{0}[\tilde{n}(\mathbf{r})]$ is the kinetic-energy functional for non-interacting electrons with density $\tilde{n}(\mathbf{r})$, and $E_{x c}[\tilde{n}(\mathbf{r})]$ is the so-called xc energy functional. $E_{x c}$ contains all many-body effects beyond the Hartree approximation, as well as the difference between the actual kinetic-energy functional of the interacting system and $T_{0}[\tilde{n}(\mathbf{r})]$. By minimising the energy functional of Eq. (2.3), one concludes that the exact density is obtained by solving self-consistently the so-called Kohn-Sham equations

$$
\left[-\frac{1}{2} \nabla^{2}+V_{e x t}(\mathbf{r})+\int d \mathbf{r}^{\prime} \frac{n\left(\mathbf{r}^{\prime}\right)}{\left|\mathbf{r}-\mathbf{r}^{\prime}\right|}+V_{x c}(\mathbf{r})\right] \phi_{i}(\mathbf{r})=\epsilon_{i} \phi_{i}(\mathbf{r}),
$$

where $V_{x c}(\mathbf{r})$ is the xc potential,

$$
V_{x c}(\mathbf{r})=\left[\frac{\delta E_{x c}[\tilde{n}(\mathbf{r})]}{\delta \tilde{n}(\mathbf{r})}\right]_{\tilde{n}(\mathbf{r})=n(\mathbf{r})}
$$

The xc energy functional is not known and must be approximated. The simplest approximation for $E_{x c}[n(\mathbf{r})]$ is the LDA,

$$
E_{x c}^{\mathrm{LDA}}[n(\mathbf{r})]=\int d \mathbf{r} n(\mathbf{r}) \epsilon_{x c}^{u n i f}(n(\mathbf{r})),
$$

where the actual xc energy per particle at point $\mathbf{r}, e_{x c}(\mathbf{r} ;[n(\tilde{\mathbf{r}})])$, which is a functional of the density $n(\tilde{\mathbf{r}})$, has been replaced by the xc energy per particle of a uniform electron gas of density $n(\mathbf{r}), \epsilon_{x c}^{u n i f}(n(\mathbf{r}))^{\mathbb{1}}$.

For an electron gas of slowly varying density the LDA may be corrected through the introduction of gradient expansions, as suggested by Kohn and Shame. Although the basic physics behind gradient expansions is correct, Langreth and Perdew showed that it fails in most practical situations because of the spurious small-wave-vector contribution to the Fourier transform of the second-order density-gradient expansion for the xc hole arounf an electron. Via cutoff of this spurious contribution, generalised gradient approximations (GGA) were then devised 12 , which approximate the xc energy per particle as a carefully chosen nonlinear function of the electron density and its gradient. Recently, a meta-generalised gradient approximation (meta-GGA) has been developed 13 , which makes use not only of the local density and its gradient but also of the orbital kinetic-energy density. For other kinds of improments of the LDA, including the weighted density approximation (WDA)14 and self-interaction corrections (SIC)15.16, we refer the reader to the review by Perdew and Kurth17.

\footnotetext{
${ }^{1}$ Several parameterisations of $\epsilon_{x c}(n)$ exist, which are based on many-body QMC simulations of the uniform electron gas 1 .
} 


\section{B. Quantum Monte Carlo methods}

Unlike the density-functional approach, in which the ground-state density is the basic quantity, wave-functionbased quantym Monte Carlo methods sample the full ground-state many-body wave function of the system under consideration 18. There are many different QMC methods, but here we will be concerned with only two of them: variational quantum Monte Carlo (VMC) and fixed-node diffusion quantum Monte Carlo (DMC), which have been shown to provide a description of increasing sophistication of the properties of zero-temperature systems.

The VMC method is based on an ansatz for a parameterised trial many-body wave function. Expectation values are evaluated by the Metropolis Monte Carlo techniques 19.20, and the free parameters are then varied in order to optimise either the energy expectation value or the fluctuation of the local energyed.

We consider trial wave functions of the Slater-Jastrow type21

$$
\Psi=D \exp \left[\sum_{i=1}^{N} \chi_{\sigma_{i}}\left(\mathbf{r}_{i}\right)-\sum_{i<j}^{N} u_{\sigma_{i}, \sigma_{j}}\left(\mathbf{r}_{i}, \mathbf{r}_{j}\right)\right],
$$

where $D$ is a Slater determinant of either Hartree-Fock (HF) or LDA single-particle orbitall 2 , $u_{\sigma_{i}, \sigma_{j}}$ is a two-body term correlating the motion of pairs of electrons, and $\sigma_{i}$ denotes the spin of electron $i$. The short-range form of $u_{\sigma_{i}, \sigma_{j}}$ is dictated by the well-known cusp condition22, while the long-range behaviour is related to the zero-point motion of plasma oscillation 23, 24. The one-body term $\chi_{\sigma_{i}}(\mathbf{r})$, which is absent in the case of a uniform electron gas, allows a variational adjustment of the electron density in the presence of the two-body term. Despite the apparent simplicity of the Slater-Jastrow trial wave function of Eq. (2.7), VMC has proved very successful in applications to bulk properties of solids. In particular, VMC methods were used to calculate cohesive energies of solid material.255 28, showing that they are typically an order of magnitude more accurate than cohesive energies obtained within the HF or LDA approaches.

The main drawback of VMC is that the accuracy of the results is entirely dependent on the quality of the trial wave function. The DMC method overcomes this limitation, by using a projection technique to enhance the ground-state component of a starting trial wave function 29 . In $\mathrm{DMC}$, the mathematical equivalence between the imaginary-time many-electron Schrödinger equation and a real-time diffusion equation is used to solve the former by a simulated numerical diffusion of a collection of fictitious particles (walkers). Each walker corresponds to a point in the $3 N$ dimensional space of electron configurations. The density of walkers represents the many-electron wave function, and the propagation of walkers, according to the imaginary-time Schrödinger equation, exponentially projects out the ground state from the initial state (provided that the initial state is not orthogonal to the ground state). In this way, an exact numerical representation of the ground state can be obtained from a reasonably accurate initial guess. The initial wave function for the more accurate and computationally expensive DMC calculation is usually provided by VMC methods.

The analogy between the imaginary-time Schrödinger equation and the diffusion equation only works if the manybody wave function is a positive (or negative) function, which can then be interpreted as a density distribution. As for fermions the antisymmetric many-electron wave function must take both positive and negative values, there is a sign problem in DMC calcylations for these particles. The common way to circumvent this problem is to use the fixed-node approximation 29.30. Within this approach, for a given trial many-electron wave function one defines a trial many-electron nodal surface on which the trial function is zero and across which it changes sign. The fixed-node DMC algorithm then produces the lowest-energy many-electron state with the given nodal surface. The resulting fixed-node errors are typically about $5 \%$ of the correlation energy (defined as the difference between the exact and the $\mathrm{HF}$ ground-state energies) 31. In most systems exchange dominates the correlation by almost an order of magnitude, and fixed-node errors are, therefore, reduced to about $0.5 \%$ of the total ground-state energy. Another possible source for systematic error in QMC calculations is the use of a finite simulation cell to model an extended system ${ }^{3}$. We shall return to these finite-size errors in section III.B, where we will examine QMC calculations of the jellium surface energy.

Although VMC and DMC methods are best suited to study the ground state, they have been shown to also provide some information about specific excited states. This subject has recently been reviewed by Foulkes et al 31 .

\footnotetext{
${ }^{2} D$ is often split into electron spin-up and spin-down Slater determinants, in order to reduce computational cost.

${ }^{3}$ Within a many-electron formulation, the Schrödinger equation cannot be solved by considering only a single primitive unit cell of the periodic nuclear potential, since the e-e interaction breaks the lattice symmetry.
} 


\section{Green-function theory}

The Kohn-Sham eigenvalues are often found to be in good agreement with the quasiparticle energies measured in photoemission experiments. Nevertheless, they have no clear physical meaning, except for the highest occupied one which corresponds to the negative of the ionisation energy 32 , and there are some serious discrepancies with measured single-particle excitation energies. Also, the knowledge of the finite lifetime of excited states is known to be outside the domain of DFT.

A prgper way of calculating single-particle excitation energies and lifetimes is provided by the Green-function theory 33 and the quasiparticle concept accounting, in a many-electron system, for the ensemble of each electron and its own xc hole. With $\mid N, s>$ as some many-body eigenstate $s$ ( $s=0$ for the ground state) of $N$ electrons, one defines the quasiparticle amplitude $\Psi_{s}(\mathbf{r})$ and the quasiparticle energy $E_{s}$ as 34

$$
\Psi_{s}(\mathbf{r})=<N, 0|\hat{\psi}(\mathbf{r})| N+1, s>, \quad E_{s}=E_{N+1,0}-E_{N, 0} \quad \text { for } \quad E_{s} \geq \mu
$$

and

$$
\Psi_{s}(\mathbf{r})=<N-1, s|\hat{\psi}(\mathbf{r})| N, 0>, \quad E_{s}=E_{N, 0}-E_{N-1, s} \quad \text { for } \quad E_{s}<\mu
$$

where $\hat{\psi}(\mathbf{r})$ is a field operator in the Heisenberg representation that destroys an electron at point $\mathbf{r}$, and $\mu$ represents the chemical potential.

The one-particle Green function 33 determines the energy and the damping of the quasiparticles. This can be shown explicitly by introducing the complete set of many-body eigenstates of the full Hamiltonian for the $N+1$ or $N-1$ particles in the definition of the one-particle Green function. One obtains

$$
G\left(\mathbf{r}, \mathbf{r}^{\prime} ; t\right)=-i \sum_{s} \Psi_{s}(\mathbf{r}) \Psi_{s}^{*}(\mathbf{r}) \mathrm{e}^{-i E_{s} t}
$$

which identifies $-2 \operatorname{Im} E_{s}$ as the inverse quasiparticle lifetime. Furthermore, Fourier transformation of Eq. (2.10) to energy space yields

$$
G\left(\mathbf{r}, \mathbf{r}^{\prime} ; E\right)=\sum_{s} \frac{\Psi_{s}(\mathbf{r}) \Psi_{s}^{*}\left(\mathbf{r}^{\prime}\right)}{E-E_{s}}
$$

which is known to satisfy the Dyson equation

$$
G\left(\mathbf{r}, \mathbf{r}^{\prime} ; E\right)=G^{0}\left(\mathbf{r}, \mathbf{r}^{\prime} ; E\right)+\int d \mathbf{r}_{1} \int d \mathbf{r}_{2} G^{0}\left(\mathbf{r}, \mathbf{r}_{1} ; E\right) \Sigma\left(\mathbf{r}_{1}, \mathbf{r}_{2} ; E\right) G\left(\mathbf{r}_{2}, \mathbf{r}^{\prime} ; E\right) .
$$

Here $G^{0}\left(\mathbf{r}, \mathbf{r}^{\prime} ; E\right)$ represents the non-interacting one-particle Green function, and $\Sigma\left(\mathbf{r}, \mathbf{r}^{\prime} ; E\right)$ is the so-called self-energy of the quasiparticle, which is a nonlocal, energy-dependent, non-Hermitian operator accounting for all xc effects beyond the Hartree approximation. By inserting the spectral representation of Eq. (2.11) into Eq. (2.12), one finds the quasiparticle equation

$$
\left[-\frac{1}{2} \nabla^{2}+V_{e x t}(\mathbf{r})+\int d \mathbf{r}^{\prime} \frac{n\left(\mathbf{r}^{\prime}\right)}{\left|\mathbf{r}-\mathbf{r}^{\prime}\right|}\right] \Psi_{s}(\mathbf{r})+\int d \mathbf{r}^{\prime} \Sigma\left(\mathbf{r}, \mathbf{r}^{\prime} ; E_{s}\right) \Psi_{s}\left(\mathbf{r}^{\prime}\right)=E_{s} \Psi_{s}(\mathbf{r}) .
$$

For a system of interacting electrons, there is little hope in solving the quasiparticle equation exactly. Hence, one usually resorts to perturbation theory and chooses, as a starting point, a suitable single-particle Hamiltonian $\hat{H}_{0}$ whose wave functions $\phi_{s}(\mathbf{r})$ and energies $\varepsilon_{s}$ approach the quasiparticle wave functions $\Psi_{s}(\mathbf{r})$ and energies $E_{s}$. In usual practice, one takes the LDA Kohn-Sham Hamiltonian

$$
\hat{H}_{0}=-\frac{1}{2} \nabla^{2}+V_{\text {ext }}(\mathbf{r})+\int d \mathbf{r}^{\prime} \frac{n\left(\mathbf{r}^{\prime}\right)}{\left|\mathbf{r}-\mathbf{r}^{\prime}\right|}+V_{x c}^{L D A}(\mathbf{r})
$$

where

$$
V_{x c}^{L D A}(\mathbf{r})=\left.\frac{d\left[n \varepsilon_{x c}^{u n i f}(n)\right]}{d n}\right|_{n=n(\mathbf{r})}
$$


The quasiparticle energy is then determined from

$$
E_{s} \approx \varepsilon_{s}+Z_{s} \Delta \Sigma_{s}\left(\varepsilon_{s}\right),
$$

where

$$
\Delta \Sigma_{s}(E)=\int d \mathbf{r} \int d \mathbf{r}^{\prime} \phi_{s}^{*}(\mathbf{r})\left[\Sigma\left(\mathbf{r}, \mathbf{r}^{\prime} ; E\right)-V_{x c}(\mathbf{r}) \delta\left(\mathbf{r}-\mathbf{r}^{\prime}\right)\right] \phi_{s}\left(\mathbf{r}^{\prime}\right)
$$

and

$$
Z_{s}=\left[1-\left.\frac{\partial \Delta \Sigma_{s}(E)}{\partial E}\right|_{E=\varepsilon_{s}}\right]^{-1}
$$

is the so-called quasiparticle weight or renormalisation factor. On the energy-shell, this factor is taken to be unity.

The exact self-energy can be obtained, in principle, from an iterative solution of Hedin's equations 34 in combination with the Dyson equation. However, to obtain explicit results one usually resorts to an expansion in powers of the timeordered screened interaction $W\left(\mathbf{r}, \mathbf{r}^{\prime} ; E\right)$. The leading-order term of this expansion is the so-called GW approximation:

$$
\Sigma\left(\mathbf{r}, \mathbf{r}^{\prime} ; E\right)=i \int_{-\infty}^{\infty} \frac{d E^{\prime}}{2 \pi} e^{-i \eta E^{\prime}} G\left(\mathbf{r}, \mathbf{r}^{\prime} ; E-E^{\prime}\right) W\left(\mathbf{r}, \mathbf{r}^{\prime} ; E^{\prime}\right),
$$

which can also be obtained as the first iteration of Hedin's equations by simply neglecting vertex corrections. The screened interaction can be expressed in terms of the density-response function $\chi\left(\mathbf{r}, \mathbf{r}^{\prime} ; E\right)$, as follows

$$
W\left(\mathbf{r}, \mathbf{r}^{\prime} ; E\right)=v\left(\mathbf{r}-\mathbf{r}^{\prime}\right)+\int \mathrm{d} \mathbf{r}_{1} \int \mathrm{d} \mathbf{r}_{2} v\left(\mathbf{r}-\mathbf{r}_{1}\right) \chi\left(\mathbf{r}_{1}, \mathbf{r}_{2}, E\right) v\left(\mathbf{r}_{2}-\mathbf{r}^{\prime}\right),
$$

where $v\left(\mathbf{r}-\mathbf{r}^{\prime}\right)$ represents the bare Coulomb interaction. If one replaces the screened interaction by $v\left(\mathbf{r}-\mathbf{r}^{\prime}\right)$ the selfenergy of Eq. (2.19) reduces to the Hartree-Fock self-energy, which sometimes leads to unphysical results, especially in the case of metals where screening plays a crucial role.

Most current GW calculations simply replace the exact one-particle Green function entering Eq. (2.19) by the non-interacting Green function $G^{0}\left(\mathbf{r}, \mathbf{r}^{\prime} ; E\right)$ pertaining to the LDA Kohn-Sham Hamiltonian of Eq. (2.14). On the same level of approximation and neglecting all vertex corrections, the screened interaction entering Eq. (2.19) is usually obtained from Eq. (2.20) with the density-response function evaluated in the random-phase approximation (RPA)円:

$$
\chi\left(\mathbf{r}, \mathbf{r}^{\prime} ; E\right)=\chi^{0}\left(\mathbf{r}, \mathbf{r}^{\prime} ; E\right)+\int \mathrm{d} \mathbf{r}_{1} \int \mathrm{d} \mathbf{r}_{2} \chi^{0}\left(\mathbf{r}, \mathbf{r}_{1} ; E\right) v\left(\mathbf{r}_{1}-\mathbf{r}_{2}\right) \chi\left(\mathbf{r}_{2}, \mathbf{r}^{\prime} ; E\right),
$$

$\chi^{0}\left(\mathbf{r}, \mathbf{r}^{\prime} ; E\right)$ being the non-interacting density-response function

$$
\chi^{0}\left(\mathbf{r}, \mathbf{r}^{\prime} ; E\right)=-2 i \int d E^{\prime} G^{0}\left(\mathbf{r}, \mathbf{r}^{\prime} ; E^{\prime}\right) G^{0}\left(\mathbf{r}, \mathbf{r}^{\prime} ; E+E^{\prime}\right) .
$$

A survey of the theory underlying the GW formalism and its applications can be found in Refs. 35 and 36. For improvements of the standard $\left(G^{0} W^{0}\right)$ quasiparticle calculations, including self-consistency and beyond-GW approximations, we refer the reader to the recent review by Aulbur, Jönsson, and Wilkins37.

\section{GROUND-STATE PROPERTIES OF SURFACES}

The simplest model to investigate the many-body properties of simple metal surfaces is the well-known jellium model. Within this model, valence electrons are described by an inhomogeneous assembly of free electrons moving in a uniform neutralising positive background, which is abruptly truncated at a plane. In this section, we focus on a description of many-body properties of jellium surfaces, and we also survey, when available, more realistic calculations where the effect of the discrete crystal lattice is taken into account. In the discussion of the jellium surface, the $z$ axis is taken to be normal to the surface, with the metal occupying the $z>0$ half-space. The positive-background charge density $\bar{n}$ is expressed in terms of the Wigner radius $r_{s}$, as $1 / \bar{n}=(4 \pi / 3) r_{s}^{3}$.

\footnotetext{
${ }^{4}$ This DFT-based RPA differs from the less realistic actual RPA, which is determined from the non-interacting Green function associated with the Hartree $\left[V_{x c}^{L D A}(\mathbf{r})=0\right.$ in Eq. (2.14)] Hamiltonian.
} 


\section{A. Pair-distribution function and exchange-correlation hole}

Central quantities in the discussion of xc effects in an interacting many-electron system are the pair-distribution function $g\left(\mathbf{r}, \mathbf{r}^{\prime}\right)$ and the xc-hole charge density $n_{x c}\left(\mathbf{r}, \mathbf{r}^{\prime}\right)$. The pair-distribution function is defined as the probability of finding an electron at point $\mathbf{r}^{\prime}$ if there is already one electron at $\mathbf{r}$

$$
g\left(\mathbf{r}, \mathbf{r}^{\prime}\right)=\frac{1}{n(\mathbf{r}) n\left(\mathbf{r}^{\prime}\right)}\left[<N, 0\left|\hat{n}(\mathbf{r}) \hat{n}\left(\mathbf{r}^{\prime}\right)\right| N, 0>-n(\mathbf{r}) \delta\left(\mathbf{r}-\mathbf{r}^{\prime}\right)\right],
$$

where $\mid N, 0>$ represents the many-body ground state of $N$ interacting electrons, $n(\mathbf{r})$ is the electron density, apd $\hat{n}(\mathbf{r})$ represents the electron-density operator. Making use of the zero-temperature fluctuation-dissipation theorem 38 , and by a suitable choice of integration contours 39 , the pair correlation function can be written in terms of the retarded density-response function $\chi\left(\mathbf{r}, \mathbf{r}^{\prime} ; E\right)$ of the many-electron system

$$
g\left(\mathbf{r}, \mathbf{r}^{\prime}\right)=1+\frac{1}{n(\mathbf{r}) n\left(\mathbf{r}^{\prime}\right)}\left[-\frac{1}{\pi} \int_{0}^{\infty} d E \chi\left(\mathbf{r}, \mathbf{r}^{\prime} ; i E\right)-n(\mathbf{r}) \delta\left(\mathbf{r}-\mathbf{r}^{\prime}\right)\right] .
$$

The total interaction energy $E_{e e}$ of the many-electron system is obtained as follows

$$
E_{e e}=\frac{1}{2} \int d \mathbf{r} \int d \mathbf{r}^{\prime} \frac{n(\mathbf{r}) g\left(\mathbf{r}, \mathbf{r}^{\prime}\right) n\left(\mathbf{r}^{\prime}\right)}{\left|\mathbf{r}-\mathbf{r}^{\prime}\right|}
$$

which can be written in the form

$$
E_{e e}=\int d \mathbf{r} n(\mathbf{r}) \varepsilon_{e e}(\mathbf{r} ;[n(\tilde{\mathbf{r}})]),
$$

$\varepsilon_{e e}(\mathbf{r} ;[n(\tilde{\mathbf{r}})])$ representing the interaction energy per particle at point $\mathbf{r}$. This interaction energy can be separated into electrostatic (Hartree) and xc terms,

$$
\varepsilon_{e e}(\mathbf{r} ;[n(\tilde{\mathbf{r}})])=\frac{1}{2} \int d \mathbf{r}^{\prime} \frac{n\left(\mathbf{r}^{\prime}\right)}{\left|\mathbf{r}-\mathbf{r}^{\prime}\right|}+\frac{1}{2} \int d \mathbf{r}^{\prime} \frac{n_{x c}\left(\mathbf{r}, \mathbf{r}^{\prime}\right)}{\left|\mathbf{r}-\mathbf{r}^{\prime}\right|}
$$

$n_{x c}\left(\mathbf{r}, \mathbf{r}^{\prime}\right)$ being the so-called xc-hole charge density

$$
n_{x c}\left(\mathbf{r}, \mathbf{r}^{\prime}\right)=n\left(\mathbf{r}^{\prime}\right)\left[1-g\left(\mathbf{r}, \mathbf{r}^{\prime}\right)\right]
$$

This quantity represents the depletion of the average electron density $n\left(\mathbf{r}^{\prime}\right)$ due to the presence of an electron at $\mathbf{r}$, which is dictated by the combined effect of the Pauli exclusion principle and Coulomb correlations, and satisfies the sum-rule

$$
\int d \mathbf{r}^{\prime} n_{x c}\left(\mathbf{r}, \mathbf{r}^{\prime}\right)=1
$$

Eqs. (3.1) (3.6) represented the starting point for regent QMC calculations of $n_{x c}$ in bulk silicon 40 , the jellium surface 142 , and strongly inhomogeneous model solid 43 . Alternatively, Eq. (3.2) allows a calculation of the pair-distribution function and the xc hole from the knowledge of the density-response function of the system. In a DFT-based RPA, this function is usually obtained from Eq. (2.21) with the use of the single-particle eigenfunctions and eigenvalues of LDA Kohn-Sham Hamiltonian of Eq. (2.14). In the framework of time-dependent densityfunctional theory 44,45 , the exact density-response function satisfies the integral equation

$$
\chi\left(\mathbf{r}, \mathbf{r}^{\prime} ; E\right)=\chi^{0}\left(\mathbf{r}, \mathbf{r}^{\prime} ; E\right)+\int d \mathbf{r}_{1} \int \mathrm{d} \mathbf{r}_{2} \chi^{0}\left(\mathbf{r}, \mathbf{r}_{1} ; E\right)\left[v\left(\mathbf{r}_{1}, \mathbf{r}_{2}\right)+f_{x c}\left(\mathbf{r}_{1}, \mathbf{r}_{2} ; E\right)\right] \chi\left(\mathbf{r}_{2}, \mathbf{r}^{\prime} ; E\right),
$$

where

\footnotetext{
${ }^{5}$ For positive energies this function coincides with its time-ordered counterpart, which enters the self-energy formalism described in the preceding section.
} 


$$
f_{x c}\left(\mathbf{r}, \mathbf{r}^{\prime} ; E\right)=\frac{\delta v_{x c}[n(\mathbf{r}, E)]}{\delta n\left(\mathbf{r}^{\prime}, E\right)}
$$

$v_{x c}[n(\mathbf{r}, E)]$ representing the exact energy-dependent xc potential, and $\chi^{0}\left(\mathbf{r}, \mathbf{r}^{\prime} ; E\right)$ being the density-response function of non-interacting Kohn-Sham electrons. In the so-called time-dependent local-density approximation (TDLDA) or, equivalently, adiabatic local-density approximation (ALDA), the Kohn-Sham effective potential is obtained in the LDA and the exact xc kernel of Eq. (3.9) is replaced, for all energies, by

$$
f_{x c}^{A L D A}\left(\mathbf{r}, \mathbf{r}^{\prime} ; E\right)=\left.\frac{d^{2}\left[n \varepsilon_{x c}^{u n i f}(n)\right]}{d n^{2}}\right|_{n=n(\mathbf{r})} \delta\left(\mathbf{r}-\mathbf{r}^{\prime}\right) .
$$

Assuming, within a jellium model of the surface, translational invariance in the plane normal to the $z$ axis, the xc-hole charge density is found to be given by the following expression:

$$
\left.n_{x c}\left(r ; z, z^{\prime}\right)=\frac{1}{2 \pi n(z)} \int d q_{\|} q_{\|} J_{0}\left(q_{\|} r\right)\left[\frac{1}{\pi} \int_{0}^{\infty} d E \chi\left(z, z^{\prime} ; q_{\|}, i E\right)\right]+n(z) \delta\left(z-z^{\prime}\right)\right],
$$

which depends on the distance $r=\left|\mathbf{r}_{\|}-\mathbf{r}_{\|}^{\prime}\right|$ projected in the plane of the surface. If the actual density-response function $\chi\left(z, z^{\prime} ; q_{\|}, i E\right)$ is replaced by its non-interacting counterpart $\chi^{0}\left(z, z^{\prime} ; q_{\|}, i E\right)$, then Eq. (3.11) yields the exchange-only or Fermi hole ${ }^{6}$.

In the simplest possible microscopic model of the jellium surface, namely the so-called infinite barrier model (IBM), the one-electron wave functions describing motion normal to the surface are simply sine functions. Using these wave functions, the exchange hole was studied by Juretschke 47 and other 48 , and the xc hole was later investigated in the RPA by Inglesfield and Moore 49 . However, the IBM does not permit the electron density to relax beyond the infinitely high potential barrier outside the surface, and yields a poor representation of both the electron density and the xc hole.

The behayipur of the exchange hole for an electron that is located well outside the surface was investigated by Sahni and BohnenE with the use of one-electron wave functions that were generated from a linear-potential model. These authors found that inside the solid, and up to the position of the jellium edge, the x-hole density $n_{x}\left(r ; z, z^{\prime}\right)$ shows a behaviour similar to that obtained within the IBM. As the electron overcomes the jellium edge, the exchange hole was found to remain behind and to be localised near the surface region, as within the IBM. However, when the electron is removed further away from the surface into the vacuum the exchange hole, instead of staying localised at the surface, was found to spread throughout the entire solid. This behaviour was further confirmed by the calculations reported by Harbola and Sahnie1, who used a step-potential model of the jellium surface. These authors also considered the $\mathrm{x}$-hole charge distribution in the plane parallel to the surface, and found that it differs significantly from the classical image charge distribution.

Recently, preliminary self-consistent calculations of both exchange and correlation contributions to the xc-hole charge density at a jellium surface were reported52, as obtained from Eqs. (3.2) and (3.6) with a full description of the DFT-based RPA density-response function of a jellium slab. These calculations showed that for an electron well outside the surface the x-only charge density displays several maxima within the solid, spaced at intervals of half a Fermi wavelength, in agreement with the results of Sahni and Bohnen, and confirmed the interesting, albeit expected result, that the build up of the image charge is entirely due to the surface-localised part of the correlation hole.

Fig. 1 shows contour plots of the fully self-consistent DFT-based RPA xc hole near a jellium surface with the positive-background charge density $\bar{n}$ of aluminium $\left(r_{s}=2.07\right)$. In the interior of the metal the xc hole is a sphere cantered at the position of the electron, as in the case of a uniform electron gas. As the electron moves towards the jellium edge, the xc-hole charge density $n_{x c}$ begins to distort and starts to lag behind. Finally, as the electron is removed far outside the surface the xc hole flattens and remains localised near the surface, becoming an image-charge distribution located at the so-called image plane.

The issue of the physical origin of the image potential has been the source of considerable controversy over the years. The asymptetic behaviour of the xc-potential $V_{x c}(\mathbf{r})$ of DFT at large distances outside a metal surface was examined by Sham 53 and by Eguiluz et al 54 through the following exact integral equation, which relates $V_{x c}(\mathbf{r})$ to the xc self-energy $\Sigma_{x c}\left(\mathbf{r}, \mathbf{r}^{\prime} ; E\right)$ 5:

\footnotetext{
${ }^{6}$ If $\chi^{0}\left(z, z^{\prime} ; q_{\|}, i E\right)$ were determined from the eigenfunctions and eigenvalues of the non-local Hartree-Fock Hamiltonian, this Fermi hole would coincide with that obtained in the Hartree-Fock approximation.
} 


$$
\int d \mathbf{r}^{\prime} V_{x c}\left(\mathbf{r}^{\prime}\right) \int d E G^{0}\left(\mathbf{r}, \mathbf{r}^{\prime} ; E\right) G\left(\mathbf{r}^{\prime}, \mathbf{r} ; E\right)=\int d \mathbf{r}_{1} \int d \mathbf{r}_{2} \int d E G^{0}\left(\mathbf{r}, \mathbf{r}_{1} ; E\right) \Sigma_{x c}\left(\mathbf{r}_{1}, \mathbf{r}_{2} ; E\right) G\left(\mathbf{r}_{2}, \mathbf{r}^{\prime} ; E\right) .
$$

Both Sham 53 and Eguiluz et al.54 concluded that the exchange self-energy $\Sigma_{x}\left(\mathbf{r}, \mathbf{r}^{\prime} ; E\right)$ yields a $1 / z^{2}$ behaviour for large $z$, while the correlation self-energy yields the image-like $1 / z$ limit, thereby confirming the long-standing believe that the image-potential structure is a pure Coulomb-correlation effect.

Harbola and Sałmi suggested an alternative procedure for constructing the xc potential that enters the Kohn-Sham equations of DFT56. These authors interpreted $V_{x c}(\mathbf{r})$ as the work required to remove an electron from the solid against the electric field of its xc-hole charge distribution, and showed that the exchange-only potential $V_{x}(\mathbf{r})$ merges with the classical image potential. Although this conclusion seemed to be in disagreement with the numerical jelliumslab $G^{0} W^{0}$ calculations reported in Ref. 54, it was then argued that while correlation gives the asymptotic image limit for slab geometries exchange does provide the limit in the case of the semi-infinite solid $\mathbb{7}$. Nevertheless, the conclusion of Harbola and Sahni56 is still in disagreement with the earlier investigations of Ref. 53, and this issue deserves further investigation. Alternatively, the image potential felt by electrons well outside the surface can be obtained from the asymptotic behaviour of the interaction energy per particle $\varepsilon_{e e}(\mathbf{r} ;[n(\tilde{\mathbf{r}})])$ of Eq. (3.5), which is dictated by the interaction between each electron and its own xc hole. Work along these lines is now in progres 58 .

\section{B. Surface energy}

One of the surface properties most sensitive to many-body effects is the surface energy. This static physical quantity is defined as the energy per unit area required to split the solid into two separate halves along a plane, i.e.,

$$
\sigma=\frac{2 E[n(\mathbf{r})]-E\left[n^{\prime}(\mathbf{r})\right]}{2 A},
$$

where $n(\mathbf{r})$ represents the ground-state electron-density distribution for each half of the solid after it is split, and $n^{\prime}(\mathbf{r})$ is that for the unsplit solid. Following the DFT energy-functional partitioning of Eq. (2.3), the surface energy can be written as the sum of three terms:

$$
\sigma=\sigma_{s}+\sigma_{e s}+\sigma_{x c}
$$

where $\sigma_{s}$ represents the kinetic surface energy of non-interacting Kohn-Sham electrons, $\sigma_{e s}$ is the electrostatic surface energy due to all positive and negative charge distributions in the solid, and $\sigma_{x c}$ is the xc contribution to the surface energy. For a jellium surface, one writes

$$
\sigma_{x c}=\int_{0}^{\infty} d z n(z)\left\{\varepsilon_{x c}(z ;[n(\tilde{z})])-\varepsilon_{x c}^{u n i f}(\bar{n})\right\},
$$

where $\varepsilon_{x c}(z ;[n(\tilde{z})])$ is the xc energy per particle at point $z$ and $\varepsilon_{x c}^{u n i f}(\bar{n})$ is the xc energy per particle of a uniform electron gas of density $\bar{n}$. In the LDA, $\varepsilon_{x c}(z ;[n(\tilde{z})])$ is simply replaced by $\varepsilon_{x c}^{u n i f}(n(z))$.

The first self-consistent DFT calculation of the jellium surface energy was carried out by Lang and Kohnt, in the LDA approximation. Lang and Kohn also discussed the effect of the crystal lattice, within first-order perturbation theory, and this work was later generalised to treat the discrete-lattice perturbation variationally 59 . Nowadays, the full inclusion, within the LDA, of lattice effects poses no difficulties; however, the question of the impact of nonlocal $\mathrm{xc}$ effects on the surface energy, and their interplay with the strong charge inhomogeneity at the surface, has remained unsettled over the years.

The original discussion about the effect-of nonlocal Coulomb correlations was centred around the surface-plasmon contribution to the surface energy. Craig 60 and Schmidt and Lucas 61 independently claimed that the surface energy of simple metals can be accounted for by the change of zero-point energy of the plasma oscillations resulting from the cleavage of the metal, which is a nonlocal effect not contained in the LDAcalculation of Lang and Kohn. However, the actual magnitude of this contribution became a matter of controversy 62 , and more careful calculations were then presented. The xc surface energy was first obtained in the RPA for a free-electron gas that was terminated abruptly at a plane 63.64, and more elaborate RPA calculations were then reported for the case in which the surface potential is taken to be an infinite barrier65. A comparison between these IBM-based RPA calculations and a local-density calculation of $\sigma_{x c}$ for the IBM density profile yielded a difference of $\sim 10 \%$, though much larger differences were found for the separate exchange and correlation contributions to $\sigma_{x c} 66$. Lang and Sham then argued that a regular power series in density gradients does not exist for separate exchange and correlation energies, and showed that the LDA is satisfactory for the combined xc term but not for exchange and for correlation separately 67 . 
RPA and ALDA calculations of jellium surface energies that are based on a fully self-consistent description of the one-particle Hartree or LDA orbitals have been performed only very recently 68 69. In these calculations, both $\varepsilon_{x c}(z ;[n(\tilde{z})])$ and $\varepsilon^{u n i f}(\bar{n})$ were obtained from a coupling-constant integration of the xc contribution to the interaction energy of Eq. (3.5) 70.71 ,

$$
\varepsilon_{x c}(\mathbf{r} ;[n(\tilde{\mathbf{r}})])=\frac{1}{2} \int_{0}^{1} d \lambda \int d \mathbf{r}^{\prime} \frac{n_{x c}^{\lambda}\left(\mathbf{r}, \mathbf{r}^{\prime}\right)}{\left|\mathbf{r}-\mathbf{r}^{\prime}\right|}
$$

where $n_{x c}^{\lambda}\left(\mathbf{r}, \mathbf{r}^{\prime}\right)$ is the xc-hole charge density of a fictitious system so chosen that as the e-e interaction strength $\lambda$ is varied in the interval $[0,1]$ the corresponding density equals its fully interacting value, i.e., $n_{\lambda}(\mathbf{r})=n(\mathbf{r})$. In Refs. 68 and 69, LDA surface energies were also computed, as obtained from the RPA and ALDA xc energies per particle of a uniform electron gas, and a comparison of local versus nonlocal surface energies supported the conclusion that the error introduced by the local-density approximation is small.

The RPA is exact for exchange and long-range eprrelations, but it is known to be a poor approximation for short-range correlations. Hence, Kurth and Perdew 72 constructed a GGA for the short-range correlation energy in combination with the DFT-based RPA calculations of Refs. 68 and 69 for long-range correlation, and obtained xc surface energies that are larger than the LDA prediction by about $2 \%$. Furthermore, it was argued that while the RPA is not a good approximation for the total correlation energy, it seems to be a surprisingly good approximation for certain changes in the correlation energy, such as those arising in surface energies 73 .

Two new approaches have been developed recently, which yield surface energies agreeing to within $1 \%$ with the so-called RPA+ calculations of Ref. 72. These are: (a) a meta-GGA13, and (b) a new wave-vector interpolation as a long-range correction to the GGA exchange and correlation 74 . Nonetheless, these calculations strongly disagree with the result of wave-function-based Fermi-hypernetted-chain (FHNC) 75 and DMC41, 42 calculations, which predict surface energies that are significantly higher than those obtained in the local-density approximation.

Acioli and Ceperley 42 performed fixed-node DMC calculations of the surface energy for a jellium slab that is contained in a finite simulation cell. They obtained surface energies that are systematically larger than the corresponding LDA results. Although for a high-density metal $\left(r_{s}=2.07\right)$ the DMC surface energy was found to be close to the LDA prediction, the difference with the LDA surface energy was found to be as large as $50 \%$ for $r_{s}=4$, in agreement with the FHNC calculations of Krotscheck and Kohn75 (the absolute difference between DMC surface energies and those obtained in the LDA was found to be of about $150 \mathrm{erg} / \mathrm{cm}^{2}$ for all densities under study, i.e., $\left.2.07 \leq r_{s} \leq 4\right)$. The discrepancy between DFT and wave-function-based surface energies is surprising: while fixed-node DMC colculatipps are often regarded as essentially exact, all the current DFT-based estimates of the jellium surface energy $13.68 .69,254$ seem to be consistent with the conclusion that LPA and GGA calculations for real surfaces provide energies at least as accurate as those derived from the experiment 7678 .

A possible source of systematic error in QMC calculations for extended systems is the use of a finite simulation cell. Hence, one needs to extrapolate the ground-state energy $E_{\infty}$ of the extended medium from that obtained for the $N$-particle system, $E_{N}$. In the case of a uniform system, one usually uses the extrapolation formula

$$
E_{\infty}=E_{N}+a\left(T_{\infty}-T_{N}\right)+\frac{b}{N}
$$

where the parameters $a$ and $b$ are fitted by considering the energy of increasingly larger systems. $T_{\infty}$ and $T_{N}$ represent the kinetic energies of the non-interacting infinite and finite systems, and the $b / N$ term accounts for the so-called Coulomb finite-size effects arising from the e-e interaction-energy term. The non-interacting term $\left(T_{\infty}-T_{N}\right)$ is generally obtained with the use of one-particle LDA calculations, and the parameter $a(a \sim 1)$ accounts for the difference between the kinetic energies of the interacting and the non-interacting systems. For non-uniform systems, there are also finite-size errors due to the electrostatic-energy contribution to the ground-state energy, which only depend on the electron density. As the LDA densities are found to be rather accurate, these finite-size errors can also be estimated within the LDA.

Acioli and Ceperly 42 estimated the finite-size corrections to their DMC surface energies from the difference between the LDA surface energies of their finite simulation cell and a jellium slab that is infinitely extended in the plane parallel to the surface. Although this estimate accounts for kinetic and (part of) electrostatic contributions to the finite-size effects, there are additional (Coulomb) finite-size errors, not encountered in the LDA, which are originated in the periodic Ewald interaction used to model the e-e Coulomb interaction in a periodic geometry.

In order to estimate the magnitude of the Coulomb finite-size corrections, which are not included in the DMC surface energies of Ref. 42, we used the VMC method to calculate the surface energy of a jellium slab with $r_{s}=3.25$. For the trial wave function we used the standard Slater-Jastrow form of Eq. (2.7) in combination with the one-body term $\chi_{i}(\mathbf{r})$ due to Fahy et al. 99 . The calculations were performed with the use of a faced-cantered cubic (fcc) supercell containing $N=284$ spin-unpolarised electrons. We modelled the e-e interaction energy using either the standard 
Ewald interaction, as used by Acioli and Ceperley 22 , or the model periodic Coulomb (MPC) interaction of Ref. 80, which virtually eliminates Coulomb finite-size effects. Our calculations indicate that for $N=284$ and $r_{s}=3.25$ Coulomb finite-size corrections to the VMC simulations are positive and amount to $3.9 \%$ of the surface energy. Since these corrections decay as $1 / N$ with the size of the system, we estimate that Coulomb finite-size corrections to the DMC calculations of Acioli and Ceperley amount to $\sim 6 \%$ of the jellium surface energy for $r_{s}=3.25$. Our preliminary VMC calculations yield surface energies of 433 and $450 \mathrm{erg} / \mathrm{cm}^{2}$, respectively, depending on whether the Ewald or the MPC interaction is employed. These surface energies are higher than predicted by the DMC calculations of Acioli and Ceperley, which yield for $r_{s}=3.25$ a surface energy of $360 \mathrm{erg} / \mathrm{cm}^{2}$, and are well above the LDA prediction of $220 \mathrm{erg} / \mathrm{cm}^{2}$ for this electron density].

Hence, finite-size corrections do not seem to account for the existing large differences between QMC and LDA surface energies, which are not present in DFT-based calculations, and a satisfactory understanding of nonlocal correlation effects in the surface energy of simple metals remains to be given in the future. Although the LDA and GGA for real metals have been found to provide surface energies in fair agreement with the experiment, this might well be due to a cancellation between the errors introduced by the LDA in the total energy of both the infinite and the semi-infinite solid. Such a cancellation of errors could not occur in the case of jellium surface energies, as the LDA is exact for the infinitely extended jellium.

\section{Work function}

The work function $\Phi$ is the minimum energy required to remove an electron from the solid to a macroscopic distance outside the surface. In a many-body context (and at $T=0$ ) this corresponds to a process in which a many-body system composed of $N$ electrons is brought from its ground state to an excited state with $N-1$ electrons in the lowest possible state in the metal and one electron at rest at infinity. Hence, one writes

$$
\Phi=E_{N^{\prime}}-E_{N}
$$

where $E_{N}$ is the ground-state energy of the semi-infinite solid and $E_{N^{\prime}}$ is the energy of the excited state. The longrange image potential tail of the surface-electron interaction potential ensures that the electron remains bounded to the surface, occupying a bound state $n$ ( $n=1$ for the lowest-lying state) of the image potential with energy

$$
\epsilon_{n}=\phi_{\infty}-\frac{1}{4(n+a)^{2}}
$$

where $a$ is the so-called quantum defect, and $\phi_{\infty}$ is the electrostatic potential well outside the surface. Thus, the minimum energy of an electron moving freely at a macroscopic distance outside the surface is

$$
\begin{aligned}
\epsilon & =\lim _{n \rightarrow \infty}\left[\phi_{\infty}-\frac{1}{4(n+a)^{2}}\right] \\
& =\phi_{\infty},
\end{aligned}
$$

and the total energy of the lowest-lying excited state of the semi-infinite solid with one electron at infinity is

$$
E_{N}^{\prime}=E_{N-1}+\phi_{\infty}
$$

As the highest occupied DFT eigenvalue $E_{F}$ equals the (negative of) the ionisation energy $E_{N}-E_{N-1}$, Eq. (3.18) may be written in the equivalent one-body form

$$
\Phi=\phi_{\infty}-E_{F}
$$

or, equivalently,

$$
\Phi=\Delta \phi-\bar{E}_{F}
$$

\footnotetext{
${ }^{7}$ In these calculations we used the DMC total energy of as quoted by Acioli and Ceperley for the total energy of the uniform electron gas. Using our own VMC energy of a uniform electron gas calculated for a system with $N=284$ electrons yields $\sigma=338 \mathrm{erg} / \mathrm{cm}^{2}$, which still is much larger than the LDA surface energy.
} 
where $\Delta \phi$ is the so-called surface dipole barrier, and $\bar{E}_{F}$ represents the Fermi level relative to the mean interior electrostatic potential.

For a jellium surface $\theta$,

$$
\bar{E}_{F}=\frac{1}{2} k_{F}^{2}+\mu_{x c}(\bar{n})
$$

where $\mu_{x c}(\bar{n})$ represents the xc potential of the uniform electron gas, and $k_{F}=\left(3 \pi^{2} \bar{n}\right)^{1 / 3}$ is the Fermi momentum. Using Eq. (3.24) one then obtains an exact general expression for the jellium work function:

$$
\Phi_{J}=\Delta \phi-\frac{1}{2} k_{F}^{2}-\mu_{x c}(\bar{n})
$$

Both $k_{F}$ and $\mu_{x c}(\bar{n})$ are known properties of the uniform electron gas and one only needs, therefore, to approximate the dipole barrier, which is usually evaluated in the LDA.

LDA self-consistent calculations of the work function of a jellium surface were carried out by Lang and Kohn 81 . The LDA xc potential decays exponentially outside the jellium surface and does not reproduce, therefore, the correct image-like asymptotic behaviour. Hence, LDA orbitals yield a too rapidly varying electron-density profile and values of the dipole barrier and the work function that are to high. Nonlocal calculations of the work function have been performed with the use of wave-function-based FHNC75 and DMC世2 methods, and they indicate that the actual work function of jellium surfaces lies about $0.3-0.5 \mathrm{eV}$ lower than their LDA counterparts. Nevertheless, an accurate determination of DMC work functions still requires longer runs and thicker simulation slabs, in order to eliminate the statistical fluctuations in the electron-density profiles and to avoid the presence of considerable finite-size effects.

In the case of real surfaces the work function is obtained from Eq. (3.23), and one needs to determine both the dipole barrier and the Fermi level $\bar{E}_{F}$. Now the bulk properties are not those of a uniform electron gas and $\bar{E}_{F}$ also needs to be approximated. In actual first-principles calculations of the work function both $\Delta \phi$ and $\bar{E}_{F}$ are usually obtained within the LDA. As the LDA uncertainties introduced in the evaluation of $\bar{E}_{F}$ tend to cancel out the corresponding uncertainties introduced in the evaluation of the dipole barrier, the LDA is expected to perform better in the evaluation of the work function of real solids than in the case of jellium. Indeed, LDA work functions of a variety of real solids have been shown to be in good agreement with experiment. This trend is particularly striking in the case of $4 d$ metals, where the relative difference between first-principles LDA and experimental work functions is found to be within $5 \%$. This is shown in Table 1

\section{QUASIPARTICLE CALCULATIONS}

\section{A. The surface barrier}

The form of the surface barrier outside a solid is of great importanfe for the interpretation of a variety of surface sensitive experiments, such as low-energy electron diffraction (LEED) 83 , scanning tunnelling microscopy (STM) 84.85 , and inverse and two-photon photoemission spectroscopy 86.87 . This issue was addressed in Refs. 53, 54, 56, 57 through the exact xc potential of DFT, i.e., the xc potential felt by Kohn-Sham electrons. A way of calculating the actual surface barrier felt by quasiparticle states of the many-body system is provided by the Green-function theory and, in particular, the nonlocal state-derpendent electron self-energy $\Sigma\left(\mathbf{r}, \mathbf{r}^{\prime} ; E\right)$.

Deisz et al. 88 and White et al.89 simulated the physics of the self-energy near the surface in terms of an effective statedependent local potential, whose real part was interpreted as the actual potential felt by a given quasiparticle state. Comparing the nonlocal Hamiltonian entering Eq. (2.13) with an effective local Hamiltonian, this state-dependent effective local xc potential was defined by

$$
V_{l o c}(\mathbf{r}) \Psi_{s}(\mathbf{r})=\int d \mathbf{r}^{\prime} \Sigma\left(\mathbf{r}, \mathbf{r}^{\prime}, E_{s}\right) \Psi_{s}\left(\mathbf{r}^{\prime}\right) .
$$

Calculations of the local potential of Eq. (4.1) were reported in Ref. 88 for the lowest-lying image resonance in a jellium surface with $r_{s}=2.07$. This effective potential was found not to depend sensitively on the quasiparticle state and to nearly coincide with the xc potential of DFT obtained in Ref. 54 by solving an exact integral equation, Eq. (3.12). Nevertheless, the calculations reported in Ref. 89 for quasiparticle states at the (111) surface of real $\mathrm{Al}$, within $1.5 \mathrm{eV}$ of the vacuum energy, indicated that the relative contributions of exchange and correlation to the image potential felt by unoccupied quasiparticle states are significantly different from the corresponding contributions to the exact $V_{x c}$ of DFT. These authors showed that in the case of quasiparticle states above the Fermi level the 
exchange part of the effective xc potential of Eq. (4.1) displays exponential decay rather than power-law behaviour, and concluded that even for a semi-infinite metal inclusion of correlation is essential for the correct description of the image potential felt by unoccupied states.

\section{B. Image-potential states}

An electron outside a metal surface can be trapped by the image-potential induced surface barrier, if its energy lies below the vacuum level and is reflected from the metal due to the lack of allowed bulk states along certain symmetry directions. This situation occurs when for a given wave vector parallel to the surface $\mathbf{k}_{\|}$there is a gap in the projected bulk band structure. Noble and transition metals usually present a surface band gap near the vacuum level $E_{v}$, and the long-range character of the image potential then gives rise to a Rydberg-like series of bound image-potential induced states whose binding energies converge for $\mathbf{k}_{\|}=0$ towards $E_{v}$ as dictated by Eq. (3.19). In the absence of a band gap, these image-states often persist as image resonances.

More than two decades ago, Echenique and Pendry 5 carried out a theoretical investigation of the integrity of image states at metal surfaces by viewing these surface states as standing waves of an electron bouncing back and forth between the semi-infinite solid and the surface barrier. Image states at metal surfaces were first detected experimentally by LEED fine-structure analysis and then by inverse photoemission (IP) 86 . 90 . 11 and two-photon photoemission (2PPE) 92 24. Although the Kohn-Sham eigenfunctions and eigenvalues have no clear physical meaning, model xc potentials that go beyond the LDA have been used to reproduce, within PFT the experimentally observed binding energies and effective masses of image states on a variety of metal surfaces 9598 .

The finite lifetime of image states at metal surfaces, which is known to be mainly due to electronic excitations in the solid 99 , is inherently outside the realm of DFT. In the framework of Green-function theory, one identifies the inverse quasiparticle lifetime as follows

$$
\tau_{s}^{-1}=-2 \operatorname{Im} E_{s},
$$

where $E_{s}$ is the quasiparticle energy. Assuming that the eigenfunctions $\phi_{s}(\mathbf{r})$ and eigenvalues $\varepsilon_{s}$ of a suitable hermitian single-particle Hamiltonian $\hat{H}_{0}$ approach the actual quasiparticle wave functions and energies, $E_{s}$ can be approximately determined from Eq. (2.16). Introduction of Eq. (2.16) into Eq. (4.2) then yields, on the energy-shell $\left(Z_{s}=1\right)$,

$$
\tau_{s}^{-1}=-2 \int d \mathbf{r} \int d \mathbf{r}^{\prime} \phi_{s}^{*}(\mathbf{r}) \operatorname{Im} \Sigma\left(\mathbf{r}, \mathbf{r}^{\prime} ; \varepsilon_{s}\right) \phi_{s}(\mathbf{r}) .
$$

If one further assumes translational invariance in the plane of the surface, single-particle wave functions are of the form

$$
\phi_{s}(\mathbf{r})=\frac{1}{\sqrt{A}} \phi_{s}(z) \mathrm{e}^{i \mathbf{k}_{\|} \cdot \mathbf{r}_{\|}},
$$

and the inverse quasiparticle lifetime is obtained as follows

$$
\tau_{s}^{-1}=-2 \int d z \int d z^{\prime} \phi_{s}^{*}(z) \operatorname{Im} \Sigma\left(z, z^{\prime} ; \mathbf{k}_{\|}, \varepsilon_{s}\right) \phi_{s}(z),
$$

where $\phi_{s}(z)$ and $\varepsilon_{s}$ now are image-state wave functions and energies describing motion normal to the surface, and $A$ represents the normalisation area.

The first quantifative evaluation of the lifetime of image states, as obtained from Eq. (4.5), was reported by Echenique et al.100. In this calculation, the image-state wave functions $\phi_{s}(z)$ were approximated by hydrogeniclike wave functions with no penetration into the solid and a simplified free-electron-gas (FEG) model was used to approximate the electron self-energy. In subsequent calculations the penetration of the image-state wave function into the crystal was allowed 101, thereby accounting for this new decay channel. The role that the narrow crystal-induced Shockley surface state on the (111) surfaces of $\mathrm{Cu}$ and Ni plays in the decay of the $n=1$ image state on these surfaces was investigated by Gao and Lundqvist102. In this work, the image-state wave functions were also approximated by hydrogenic-like wave functions with no penetration into the solid, a simplified parameterised form was used for the Shockley surface-state wave function, and screening effects were neglected altogether.

The first self-consistent many-body calculations of image-state lifetimes on noble and simplemetals were reported only very recently 103 , and good agreement with experimentally determined decay times 104 100 was found. In Ref. 103, the decay rate of image states was computed from Eq. (4.5) with the electron self-energy evaluated in the $G^{0} W^{0}$ 
approximation. The image-state wave function $\phi_{s}(z)$ and energy $\varepsilon_{s}$ and all the eigenfunctions and eigenvalues entering the non-interacting Green function $G^{0}\left(z, z^{\prime} ; \mathbf{k}_{\|}, \varepsilon\right)$ were obtained by solving a one-particle Schrödinger equation with a realistic one-dimensional model potential, and the potential variation in the plane parallel to the surface was considered thorugh the introduction of the effective mass.

Self-consistent calculations of the key role that the Shockley surface state plays in the decay-gf image states on $\mathrm{Cu}$ surfaces were later carried out 107 , and the inclusion of short-range xc effects was investigated 100 in the framework of the so-called $G W \Gamma$ approximation 109.10. In this approximation, the electron self-energy is of the $G W$ form, i.e., it is given by Eq. (2.19), but with an effective screened interaction

$$
W\left(\mathbf{r}, \mathbf{r}^{\prime} ; E\right)=v\left(\mathbf{r}-\mathbf{r}^{\prime}\right)+\int \mathrm{d} \mathbf{r}_{1} \int \mathrm{d} \mathbf{r}_{2}\left[v\left(\mathbf{r}-\mathbf{r}_{1}\right)+f_{x c}\left(\mathbf{r}_{1}, \mathbf{r}_{2} ; E\right)\right] \chi\left(\mathbf{r}_{1}, \mathbf{r}_{2}, E\right) v\left(\mathbf{r}_{2}-\mathbf{r}^{\prime}\right),
$$

the density-response function now being the time-ordered counterpart of the retarded density-response function of Eq. (3.8). The xc kernel $f_{x c}\left(\mathbf{r}_{1}, \mathbf{r}_{2} ; E\right)$ entering Eqs. (3.8) and (4.6) account for the reduction in the e-e interaction due to the existence of short-range xc effects associated to the image-state electron and to screening electrons, respectively. In Ref. 108 the exact xc kernel was replaced by that of Eq. (3.10), and it was concluded that $G^{0} W^{0}$ calculations produce decay rates that are within $5 \%$ of the more realistic $G^{0} W \Gamma$ calculations. Although the presence of shortrange exchange and correlation between screening electrons significantly enhances the decay probability of image states, this enhancement was found to be more than compensated by the large reduction in the decay rate produced by the presence of a xc hole around the image-state electron itself.

Unlike image states, which are unoccupied states with energies close to the vacuum level, crystal-induced Shockley surface states are known to exist near the Fermi level in the $\Gamma$-L projected bulk band gap of the (111) surfaces of the noble metals $\mathrm{Cu}, \mathrm{Ag}$, and $\mathrm{Au} 111-113$. Hence, these surface states form a quasi two-dimensional (2D) electron gas, which overlaps in energy and space with the three-dimensional (3D) substrate, and represent a promising playground for lifetime investigations.

Photohole lifetimes of Shockley surface states were investigatedin a variety of metal surfaces with the use of highresolution angle-resolved photoemission (ARP) spectroscopy 114 116. Recently, the STM was used to determine the lifetime of excited holes at the edge of the partially occupied surface-state band on the (111) face of noble metals 117 , and also to measure the lifetime of hot surface-state and surface-resonance electrons, as a function of energy 118 . $G^{0} W^{0}$ calculation.119, as obtained from Eq. (4.3), demonstrated that the decay of surface-state holes is dominated by $2 \mathrm{D}$ electron-electron interactions screened by the underlying $3 \mathrm{D}$ electron system, and showed an excellent agreement with the experiment. These theoretical investigations were then extended to the case of hot surface-state and surfaceresonance electrons in $\mathrm{Cu}(111) 120$, showing that, contrary to the case of surface-state holes, major contributions to the e-e interaction of surface-state electrons above the Fermi level come from the underlying bulk electrons and thereby giving an interpretation to the measurements reported in Ref. 118.

\section{PERSPECTIVES FOR FUTURE RESEARCH}

Approximate density-functipnal treatments of many-body effects are being applied to increasingly more complex problems in surface science 21 . The question of their accuracy in describing these effects, e.g., in chemical reactions at surfaces, and how to improve them systematically is still outstanding. In the case of a jellium surface many-body effects are not masked by the effect of the crystal structure, so this system provides an stringent test of the accuracy of approximate density functionals in strongly inhomogeneous systems. In principle, approximate density functionals, as well as other many-body approaches, could be benchmarked for the jellium surface against diffusion Monte Carlo results. However, the presence of rather large statistical fluctuations (in the electron-density profile) and finite-size errors (in the surface energy) makes it difficult to give a clear verdict on the performance of LDA, GGA, and RPA on the basis of the current DMC data. There is a clear need for new DMC calculations of the work function and the surface energy of jellium surfaces which are performed with the use of larger system sizes, longer runs, and an improved treatment of finite-size effects.

Although computationally much more expensive than DFT calculations, QMC algorithms have the important computational feature of being inherently parallel. The use of massively parallel computers has made the application of QMC methods to the investigation of real solids possible. Furthermore, QMC studies of real surfaces are now within reach, and a full-many body description of chemical reactions at surfaces appears to be an exciting perspective further down the line.

Recently, the Green-function theory, within the GW approximation, has been shown to provide total energies of an infinitely extended free-electron system that are as accurate as those obtained from DMC calculations, as long as the GW approximation is treated self-consistently 22 . Within this approach, the Green function entering the GW 
self-energy of Eq. (2.19) is evaluated self-consistently from the Dyson equation [Eq. (2.12)], the screened interaction being obtained as in the RPA through Eqs. 2.20)-2.2. - but with the non-interacting Green function $G^{0}\left(\mathbf{r}, \mathbf{r}^{\prime} ; E\right)$ replaced by its self-consistent interacting counterpart 22,123 . The total energy is then obtained from the so-called Galitskii-Migdal formula33. The application of this way of obtaining the total energy to the case of a jellium surface seems to be promising in the effort to understand the current discrepancies between DFT and wave-function-based estimates of the surface energy.

Excited-state properties are still the realm of Green function methods (in the GW approximation). In the case of image-potential induced states these methods have the great advantage of providing, within the same computational framework, a consistent description of both binding energies and lifetimes, as well as the surface barrier. So far, full GW calculations have been limited to the study of jellium surfaces and the (111) surface of Al, both of which support only image resonances. However, the binding energies and intrinsic lifetimes of these resonances are not welldefined and are difficult to compare with the experiment. On the other hand, currently available GW calculations of well-defined image states on noble metal surfaces have been carried out with the use of a model one-dimensional potential, and first-principles many-body descriptions that are based on a complete three-dimensional treatment of the band structure of the solid would be desirable. Since binding energies of image states are very sensitive to the surface barrier, comparison between these calculations and the available experimental data should also help to resolve remaining questions, such as the precise many-body origin of the image potential.

\section{ACKNOWLEDGEMENTS}

The authors would like to thank Matthew Foulkes for useful discussions in connection with this research. J.M.P. acknowledges partial support by the University of the Basque Country, the Basque Unibertsitate eta Ikerketa Saila, and the Spanish Ministerio de Educación y Cultura.

${ }^{1}$ P. Hohenberg and W. Kohn, Phys. Rev. 136, B864 (1964).

${ }^{2}$ W. Kohn and L. Sham, Phys. Rev. 140, A1133 (1965).

${ }^{3}$ For a review see J. E. Inglesfield in Cohesion and Structure of Surfaces Vol 4, edited by K. Binder, M. Bowker, J. E. Inglesfield and P. J. Rous (Elsevier, Amsterdam, 1995), p. 63).

${ }^{4}$ N. D. Lang and W. Kohn, Phys. Rev. B 1, 4555 (1970); N. D. Lang, Solid State Phys. 28, 225 (1973).

${ }^{5}$ P. M. Echenique and J. B. Pendry, J. Phys. C 11, 2065 (1978); P. M. Echenique and J. B. Pendry, Prog. Surf. Sci. 32, 111 (1990).

${ }^{6}$ D. M. Ceperley and B. J. Alder, Phys. Rev. Lett. 45, 1196 (1980).

${ }^{7}$ D. C. Langreth and J. P. Perdew, Phys. Rev. B 21, 5469 (1980).

${ }^{8}$ D. C. Langreth and M. J. Mehl, Phys. Rev. B 28, 1809 (1983).

${ }^{9}$ J. P. Perdew and Y. Wang, Phys. Rev. B 33, 8800 (1986).

${ }^{10}$ A. D. Becke, Phys. Rev. A 38, 3098 (1988).

11 J. P. Perdew, K. Burke, and M. Ernzerhof, Phys. Rev. Lett. 77, 3865 (1996).

12 A. D. Becke, J. Chem. Phys. 109, 2092 (1998).

13 J. P. Perdew, S. Kurth, A. Zupan, and P. Blaha, Phys. Rev. Lett. 82, 2544 (1999).

${ }^{14}$ O. Gunnarsson, R. O. Jones, and B. I. Lundqvist, Sol. State. Comm. 24, 765 (1977); Phys. Rev. B 20, 3136 (1979).

15 J. P. Perdew, Chem. Phys. Lett. 64, 127 (1979).

16 J. P. Perdew and A. Zunger, Phys. Rev. B 23, 5048 (1981).

17 J. P. Perdew and S. Kurth, in Density Functionals: Theory and Applications, edited by D. Joubert (Springer, Berlin, 1998), p. 8.

${ }^{18}$ D. M. Ceperley, in Recent Progress in Many-Body Theories, edited by J. G. Zabolitsky (Springer, Berlin, 1981 ), p. 262.

${ }^{19}$ N. Metropolis, A. W. Rosenbluth, M. N. Rosenbluth, A. H. Teller, and E. Teller, J. Chem. Phys. 21, 1087 (1953).

${ }^{20}$ B. L. Hammond, W. A. Lester, Jr., and P. J. Reynolds, Monte Carlo Methods in Ab Initio Quantum Chemistry (World Scientific, Singapore, 1994).

${ }^{21}$ R. J. Jastrow, Phys. Rev. 98, 1479 (1955).

22 T. Kato, Commun. Pure Appl. Math. 10, 151 (1957).

${ }^{23}$ D. Bohn and D. Pines, Phys. Rev. 92, 609 (1953).

${ }^{24}$ R. Gaudoin, M. Nekovee, W.M.C. Foulkes, R.J. Needs, and G. Rajagopal, cond-mat/0005305.

${ }^{25}$ S. Fahy, X. W. Wang, and S. G. Louie, Phys. Rev. Lett. 61, 1631 (1988); Phys. Rev. B 42, 3503 (1990). 
${ }^{26}$ X.-P. Li, D. M. Ceperley, and R. M. Martin, Phys. Rev. B 44, 10929 (1991).

${ }^{27}$ G. Rajagopal, R. J. Needs, A. James, S. D. Kenny, and W. M. C. Foulkes, Phys. Rev. B 51, 10591 (1995).

${ }^{28}$ P. R. C. Kent, R. Q. Hood, A. J. Williamson, R. J. Needs, and G. Rajagopal, Phys. Rev. B 57, 15293 (1998).

${ }^{29}$ P. J. Reynolds, D. M. Ceperley, B. J. Alder, and W. A. Lester, J. Chem. Phys. 77, 5593 (1982).

30 J. W. Moskowitz, K. E. Schmidt, M. A. Lee, and M. H. Kalos, J. Chem. Phys. 77, 349 (1982).

${ }^{31}$ W. M. C. Foulkes, L. Mitas, R. J. Needs, and G. Rajagopal, Rev. Mod. Phys. (in press).

32 C.-O. Almbladh and U. Von Barth, Phys. Rev. B 31, 3231 (1985).

${ }^{33}$ V. Galitskii and A. Migdal, Sov. Phys. JETP 7, 96 (1958).

${ }^{34}$ L. Hedin, Phys. Rev. 139 A796 (1965); L. Hedin and S. Lundqvist, Solid State Phys. 23, 1 (1969).

${ }^{35}$ F. Aryasetiawan and O. Gunnarsson, Re. Prog. Phys. 61, 237 (1998).

${ }^{36}$ B. Farid, in Electron Correlation in the Solid State, edited by N. H. March (Imperial College Press, London, 1999).

${ }^{37}$ W. G. Aulbur, L. Jönsson, and J. W. Wilkins, Solid State Phys. 54, 1 (2000).

${ }^{38}$ H. B. Callen and T. R. Welton, Phys. Rev. P83, 34 (1951).

${ }^{39}$ D. Pines, Elementary Excitations in Solids (W. A. Benjamin, New York, 1963).

${ }^{40}$ R. Q. Hood, M.-Y. Chou, A. J. Williamson, G. Rajagopal, R. J. Needs, and W. M. C. Foulkes, Phys. Rev. Lett. 78, 3350 (1997).

${ }^{41}$ X.-P. Li, R. J. Needs, R. M. Martin, and D. M. Ceperley, Phys. Rev. B 45, 6124 (1992).

${ }^{42}$ P. H. Acioli and D. M. Ceperley, Phys. Rev. B 54, 17199 (1996).

${ }^{43}$ M. Nekovee, W. M. C. Foulkes, and R. J. Needs (unpublished); M. Nekovee, W. M. C. Foulkes, G. Rajagopal, A. J. Williamson and R.J. Needs, Adv. Quantum Chem. 33, 189 (1999).

${ }^{44}$ E. Runge and E. K. U. Gross, Phys. Rev. Lett. 52, 997 (1984); E. K. U. Gross and W. Kohn, Phys. Rev. Lett. 55, 2850 (1985).

45 M. Petersilka, U. J. Gossmann, and E. K. U. Gross, Phys. Rev. Lett. 76, 1212 (1996).

46 A. Zangwill and P. Soven, Phys. Rev. A 21, 1561 (1980).

${ }^{47}$ H. J. Juretschke, Phys. Rev. 92, 1140 (1953).

${ }^{48}$ I. D. Moore and N. H. March, Ann. Phys. (N. Y.) 97, 136 (1976).

49 J. E. Inglesfield and I. D. Moore, Solid State Commun. 26, 867 (1978).

${ }^{50}$ V. Sahni and K.-P Bohnen, Phys. Rev B 29, 1045 (1984); 31, 7651 (1985).

${ }^{51}$ M. K. Harbola and V. Sahni, Phys. Rev. B 37, 745 (1988).

52 J. M. Pitarke and A. G. Eguiluz, Bull. Am. Phys. Soc. 39, 515 (1994); 40, 33 (1995).

${ }^{53}$ L. J. Sham, Phys. Rev. B 32, 3876 (1985).

54 A. G. Eguiluz, M. Heinrichsmeier, A. Fleszar, and W. Hanke, Phys. Rev. Lett. 68, 1359 (1992).

${ }^{55}$ L. J. Sham and M. Schlüter, Phys. Rev. Lett. 51, 1888 (1983).

${ }^{56}$ M. K. Harbola and V. Sahni, Phys. Rev. Lett. 62, 489 (1989).

57 A. Solomatin and V. Sahni, Ann. Phys.-New York 259, 97 (1997).

${ }^{58}$ J. M. Pitarke (unpublished).

${ }^{59}$ R. Monnier and J. P. Perdew, Phys. Rev. B 17, 2595 (1978).

${ }^{60}$ R. A. Craig, Phys. Rev. B 6, 1134 (1972).

${ }^{61}$ J. Schmit and A. A. Lucas, Solid State Commun. 26, 867 (1972).

${ }^{62}$ P. J. Feibelman, Solid State Commun. 13, 319 (1973); M. Jonson and G. Srinivasan, Phys. Lett. A 43, 427 (1973); W. Kohn, Solid State Commun. 13, 323 (1973)

${ }^{63}$ J. Harris and R. O. Jones, Phys. Lett. A 46, 407 (1974); J. Phys. F. 4, 1170 (1974).

${ }^{64}$ G. Srinivasan and M. Jonson, Solid State Commun. 15, 771 (1974); Phys. Scr. 10, 262 (1974).

${ }^{65}$ E. Wikborg and J. E. Inglesfield, Solid State Commun. 16, 335 (1975); Phys. Scr. 15, 37 (1977).

${ }^{66}$ D. C. Langreth and J. P. Perdew, Solid State Commun. 17, 1425 (1975).

${ }^{67}$ N. D. Lang and L. J. Sham, Solid State Commun. 17, 581 (1975).

${ }^{68}$ J. M. Pitarke and A. G. Eguiluz, Phys. Rev. B 57, 6329 (1998).

69 J. M. Pitarke and A. G. Eguiluz (unpublished).

${ }^{70}$ O. Gunnarsson and B. I. Lundqvist, Phys. Rev. B 13, 4274 (1976).

${ }^{71}$ D. C. Langreth and J. P. Perdew, Phys. Rev. B 15, 2884 (1977).

72 S. Kurth and J. P. Perdew, Phys. Rev. B 59, 10461 (1999).

73 Z. Yan, J. P. Perdew, and S. Kurth, Phys. Rev. B 61, 16430 (2000).

${ }^{74}$ Z. Yan, J. P. Perdew, S. Kurth, C. Fiolhais, and L. Almeida, Phys. Rev. B 61, 2595 (2000).

${ }^{75}$ E. Krotscheck and W. Kohn, Phys. Rev. Lett. 57, 862 (1986).

76 M. Methfessel, D. Henning, and M. Scheffler, Phys. Rev. B 46, 4816 (1992).

${ }^{77}$ H. L. Skriver and N. M. Rosengaard, Phys. Rev. B 46, 7157 (1992).

${ }^{78}$ L. Vitos, A. V. Ruban, H. L. Skriver, and J. Kllár, Surf. Sci. 411, 186 (1999).

${ }^{79}$ A. Malatesta, S. Fahy and G. B. Bachelet, Phys. Rev. B 56, 12201 (1997).

${ }^{80}$ L. M. Fraser, W. M. C. Foulkes, G. Rajagopal, R. J. Needs, S. D. Kenny, and A. J. Williamson, Phys. Rev. B 53, 1814 (1996); A. J. Williamson, G. Rajagopal, R. J. Needs, L. M. Fraser, W. M. C. Foulkes, Y. Wang, and M.-Y. Chou, Phys. 
Rev. B 55, R4851 (1997).

${ }^{81}$ N. D. Lang and W. Kohn, Phys. Rev. B 3, 1215 (1971).

${ }^{82}$ R. de Boer, R. Boom, W. C. M. Mattens, A. R Midema, and A. K Niessen, Cohesion in Metals (North-Holand, Amsterdam, 1988).

83 J. Rundgren and G. Malmström, Phys. Rev. Lett. 38, 836 (1977).

${ }^{84}$ G. Binnig, N. García, H. Rohrer, J. M. Soler, and F. Flores, Phys. Rev. B 30, 4816 (1984); G. Binnig, K. H. Frank, H. Fuchs, N. García, B. Reihl, H. Rohrer, F. Salvan, and A. R. Williams, Phys. Rev. Lett. 55, 991 (1985).

85 J. M. Pitarke, P. M. Echenique, and F. Flores, Surf. Sci. 217, 267 (1989); J. M. Pitarke, P. M. Echenique, and F. Flores, Surf. Sci. 234, 1 (1990).

${ }^{86}$ P. D. Johnson and N. V. Smith, Phys. Rev. B 27, 2527 (1983).

87 Th. Fauster and W. Steinmann, in Electromagnetic Waves: Recent Development in Research, Vol. 2, p. 350, P. Halevi (Ed.), Elsevier, Amsterdam, 1995.

${ }^{88}$ J. J. Deisz, A. G. Eguiluz, and W. Hanke, Phys. Rev. Lett. 71, 2793 (1993); J. Deisz and A. G. Eguiluz, J. Phys.: Condens. Matter 5, A95 (1993).

${ }^{89}$ I. D. White, R. W. Godby, M. M. Rieger, and R. J. Needs, Phys. Rev. Lett. 80, 4265 (1998).

${ }^{90}$ V. Dose, W. Altmann, A. Goldmann, U. Kolac, and J. Rogozik, Phys. Rev. Lett. 52, 1919 (1984).

${ }^{91}$ D, Straub and F. J. Himpsel, Phys. Rev. Lett. 52, 1922 (1984); Phys. Rev. B 33, 2256 (1986).

${ }^{92}$ K. Giesen, F. Hage, F. J. Himpsel, H. J. Riess, and W. Steinmann, Phys. Rev. Lett. 55, 300 (1985).

${ }^{93}$ R. W. Schoenlein, J. G. Fujimoto, G. L. Eesley, T. W. Capehart, Phys. Rev. Lett. 61, 2596 (1988); Phys. Rev. B 43, 4688 (1991).

94 S. Schuppler, N. Fischer, Th. Fauster, and W. Steinmann, Phys. Rev. B 46, 13539 (1992).

${ }^{95}$ M. Weinert, S. L. Hulbert, and P. D. Johnson, Phys. Rev. Lett. 55, 2055 (1985); S. L. Hulbert, P. D. Johnson, M. Weinert, and R. F. Garrett, Phys. Rev. B 33, 760 (1986).

${ }^{96}$ N. V. Smith, C. T. Chen, and M. Weinert, Phys. Rev. B 40, 7565 (1989).

${ }^{97}$ M. Nekovee and J. E. Inglesfield, Europhys. Lett. 19, 535 (1992).

${ }^{98}$ M. Nekovee, S. Crampin, and J. E. Inglesfield, Phys. Rev. Lett. 70, 3099 (1993).

${ }^{99}$ P. M. Echenique, J. M. Pitarke, E. V. Chulkov, and A. Rubio, Chem. Phys. 251, 1 (2000).

100 P. M. Echenique, F. Flores, and F. Sols, Phys. Rev. Lett. 55, 2348 (1985).

${ }^{101}$ P. L. de Andrés, P. M. Echenique, an F. Flores, Phys. Rev. B 35, 4529 (1987); Phys. Rev. B 39, 10356 (1989).

102 S. Gao and B. I. Lundqvist, Prog. Theor. Phys. Suppl. 106, 405 (1991); S. Gao and B. I. Lundqvist, Solid State Commun. 84, 147 (1992).

${ }^{103}$ E. V. Chulkov, I. Sarria, V. M. Silkin, J. M. Pitarke, and P. M. Echenique, Phys. Rev. Lett. 80, 4947 (1998); E. V. Chulkov, J. Osma, I. Sarria, V. M. Silkin, and J. M. Pitarke, Surf. Sci. 433, 882 (1999).

${ }^{104}$ M. Wolf, E. Knoesel, and T. Hertel, Phys. Rev. B 54, 5295 (1997); M. Wolf, Surf. Sci. 377, 343 (1997).

${ }^{105}$ E. Knoesel, A. Hotzel, and M. Wolf, J. Electron. Spectrosc. Relat. Phenom. 88, 577 (1998).

${ }^{106}$ U. Höfer, I. L. Shumay, Ch. Reuss, U. Thomann, W. Wallauer, and Th. Fauster, Science 277, 1480 (1997); I. L. Shumay, U. Höfer, Ch. Reuss, U. Thomann, W. Wallauer, and Th. Fauster, Phys. Rev. B 58, 13974 (1998).

107 J. Osma, I. Sarria, E. V. Chulkov, J. M. Pitarke, and P. M. Echenique, Phys. Rev. B 59, 10591 (1999).

${ }^{108}$ I. Sarria, J. Osma, E. V. Chulkov, J. M. Pitarke, and P. M. Echenique, Phys. Rev. B 60, 11795 (1999).

109 T. M. Rice, Ann. Phys. (N.Y.) 31, 100 (1965).

${ }^{110}$ G. D. Mahan and B. Sernelius, Phys. Rev. Lett. 62, 2718 (1989); G. D. Mahan, Many-Particle Physics, 2nd ed. (Plenum, New York, 1990).

${ }^{111}$ P. O. Gartland and B. J. Slagsvold, Phys. Rev. B 12, 4047 (1977).

112 W. Eberhardt and E. W. Plummer, Phys. Rev. B 21, 3245 (1980).

${ }^{113}$ F. J. Himpsel, Comments. Cond. Matter Phys. 12, 199 (1986).

114 S. D. Kevan, Phys. Rev. Lett. 50, 526 (1983); J. Tersoff, S. D. Kevan, Phys. Rev. B 28, 4267 (1983).

${ }^{115}$ F. Theilmann, R. Matzdorf, G. Meister, and A. Goldmann, Phys. Rev. B 56, 3632 (1997).

116 T. Balasubramanian, E. Jensen, X. L. Wu, and S. L. Hulbert, Phys. Rev. B 57, R6866 (1998).

117 J. Li., W.-D. Schneider, R. Berndt, O. R. Bryant, and S. Crampin, Phys. Rev. Lett. 81, 4464 (1998).

${ }^{118}$ L. Bürgi, O. Jeandupeux, H. Brune, and K. Kern, Phys. Rev. Lett. 82, 4516 (1999).

119 J. Kliewer, R. Berndt, E. V. Chulkov, V. M. Silkin, P. M. Echenique, and S. Crampin, Science 288, 1399 (2000); P. M. Echenique, J. Osma, V. M. Silkin, E. V. Chulkov, and J. M. Pitarke, Appl. Phys. A (in press).

${ }^{120}$ P. M. Echenique, J. Osma, M. Machado, V. M. Silkin, E. V. Chulkov, and J. M. Pitarke, Prog. Surf. Sci. (in press).

${ }^{121}$ See, e.g, B. Hammer and J. K. Norskov, Adv. in Catalysis 4545 (2000), D. M. Bird and P. A Gravil, Surf. Science 377, 555 (1997).

${ }^{122}$ B. Holm and U. von Barth, Phys. Rev. B 57, 2108 (1998); B. Holm, Phys. Rev. Lett. 83, 788 (1999).

${ }^{123}$ W. D. Schöne and A. G. Eguiluz, Phys. Rev. Lett. 81, 1662 (1998). 
TABLF- LDA and experimental values of the work function for low-index crystal faces of $4 d$ metals, taken from ${ }^{a}$ Ref $\frac{76}{3}$ and ${ }^{b}$ Ref. ${ }^{82}$, respectively
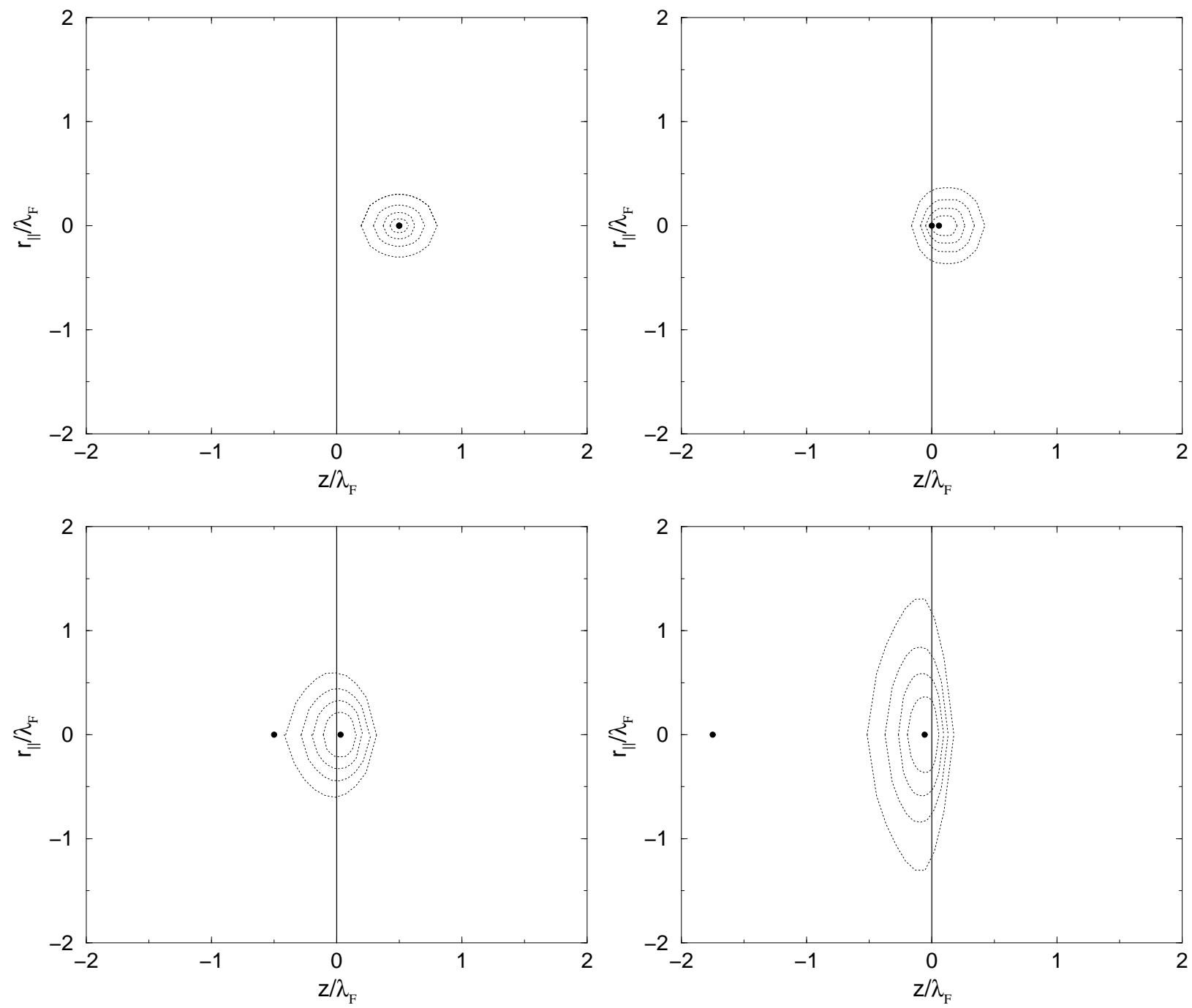

FIG. 1. Contour plots of exchange-correlation hole for jellium surface for $r_{s}=2.07$ calculated within the DFT-based RPA. Snapshots show deformation of the hole around the electron as the electron moves from a point well inside metal to the vacuum. The vertical line represents the edge of the positive background. One of the circles represent the position of the electron. The other circle represent the centre of gravity of the hole. Metal occupies $z \geq 0$. 